\title{
ICU-acquired weakness: what is preventing its rehabilitation in critically ill patients?
}

Christie M Lee ${ }^{1,2}$ and Eddy Fan ${ }^{1 *}$

\begin{abstract}
Intensive care unit-acquired weakness (ICUAW) has been recognized as an important and persistent complication in survivors of critical illness. The absence of a consistent nomenclature and diagnostic criteria for ICUAW has made research in this area challenging. Although many risk factors have been identified, the data supporting their direct association have been controversial. Presently, there is a growing body of literature supporting the utility and benefit of early mobility in reducing the morbidity from ICUAW, but few centers have adopted this into their ICU procedures. Ultimately, the implementation of such a strategy would require a shift in the knowledge and culture within the ICU, and may be facilitated by novel technology and patient care strategies. The purpose of this article is to briefly review the diagnosis, risk factors, and management of ICUAW, and to discuss some of the barriers and novel treatments to improve outcomes for our ICU survivors.
\end{abstract}

Keywords: critical illness, early ambulation, extracorporeal membrane oxygenation, intensive care units, muscle weakness, physical therapy, rehabilitation

\section{Background}

Decreasing mortality following critical illness over the last few decades has shifted the focus from short-term to long-term outcomes in intensive care unit (ICU) survivors. Survivors are often left with profound neuromuscular weakness resulting in persistent functional disability and decreased quality of life for years after hospital discharge [1,2]. Reasons for the development of muscle weakness are multifactorial, including premorbid

\footnotetext{
* Correspondence: efan@mtsinai.on.ca

${ }^{1}$ Interdepartmental Division of Critical Care Medicine, University of Toronto, and the Division of Respirology, Department of Medicine, Mount Sinai Hospital and University Health Network, Toronto, Ontario, Canada Full list of author information is available at the end of the article
}

conditions (for example, malignancy) and ICU-acquired weakness (ICUAW) that may develop as a result of critical illness, systemic inflammation, and certain ICU exposures (for example, enforced bed rest) [3]. This article will focus on reviewing some of the more commonly perceived causes of ICU-acquired weakness, including hyperglycemia, exposure to corticosteroids, and the use of neuromuscular blockers. Currently, no specific treatment exists for ICUAW. Over the last decade, a number of studies have demonstrated the safety and success of early rehabilitation in the ICU [4-6], which may be a promising therapy for the prevention of ICUAW. However, a number of important barriers to advancing our knowledge of the etiology, mechanisms, prevention, and treatment of ICUAW continue to exist. We will discuss some of the issues surrounding early mobility in the ICU, barriers to the implementation of early mobility, and finally, discuss some novel ventilatory strategies to facilitate an awake, calm, and mobile patient in the ICU.

\section{ICUAW: a rose by any other name}

The first reported cases of flaccid paralysis from peripheral neuropathy following sepsis was documented by Bolton et al., in 1984 in patients unable to wean from mechanical ventilation [7]. Since then, numerous case series and observational studies of ICUAW have led to an explosion in the number of terms used to describe this syndrome [8]. Concomitantly, there has been an evolving understanding into the various mechanisms by which ICUAW develops, and potential therapeutic targets (for example, physical therapy) for this syndrome, which have been recently reviewed $[3,9,10]$. However, the absence of a consistent nomenclature has made research in this area challenging, in particular, with two systematic reviews revealing major differences in the definition, diagnosis, reporting of outcomes, and risk factors associated with this syndrome [11,12]. As a result, a new scheme to diagnose and classify ICUAW has been proposed [8]. Broad consensus by clinicians and researchers on a common definition and diagnostic 
Table 1 Clinical, electrophysiological, and histological features of intensive care unit-acquired weakness (ICUAW)

\begin{tabular}{|c|c|c|c|}
\hline Investigation & CIP & CIM & CINM \\
\hline \multirow{3}{*}{$\begin{array}{l}\text { Physical } \\
\text { examination }\end{array}$} & Distal muscle weakness & Proximal muscle weakness & Proximal and distal muscle weakness \\
\hline & Distal sensory deficit & Normal sensory testing & Distal sensory deficit \\
\hline & $\begin{array}{l}\text { Normal or depressed deep } \\
\text { tendon reflexes }\end{array}$ & $\begin{array}{l}\text { Normal or depressed deep tendon } \\
\text { reflexes }\end{array}$ & Depressed deep tendon reflexes \\
\hline \multirow[t]{3}{*}{$\begin{array}{l}\text { Electrophysiology } \\
\text { studies }\end{array}$} & $\begin{array}{l}\text { Decreased CMAP and decreased } \\
\text { SNAP }\end{array}$ & Decreased CMAP and normal SNAP & Decreased CMAP and SNAP \\
\hline & Normal MUAP & Decreased MUAP & Decreased MUAP \\
\hline & $\begin{array}{l}\text { Normal or near-normal } \\
\text { conduction velocity }\end{array}$ & $\begin{array}{l}\text { EMG shows short duration, low } \\
\text { amplitude activity }\end{array}$ & EMG shows short duration, low-amplitude activity \\
\hline Histology & $\begin{array}{l}\text { Axonal degeneration of distal } \\
\text { motor and sensory nerves }\end{array}$ & $\begin{array}{l}\text { Thick filament (myosin) loss, type II fiber } \\
\text { (fast twitch) atrophy, necrosis }\end{array}$ & $\begin{array}{l}\text { Axonal degeneration and evidence of loss in } \\
\text { myosin, type II fiber atrophy, and necrosis }\end{array}$ \\
\hline
\end{tabular}

$\mathrm{CIM}=$ critical illness myopathy; CINM = critical illness neuromyopathy; CIP = critical illness polyneuropathy; CMAP = compound muscle action potential; EMG = electromyography; MUAP = muscle unit action potential; SNAP= sensory nerve action potential.

criteria for ICUAW would ensure consistent identification of ICUAW patients and the ability to compare results across studies and patient populations. Ultimately, the diagnosis should begin with routine physical examination of a patient that is weak following an episode of critical illness (Table 1). An initial examination should be performed upon admission to the ICU, with subsequent examinations paired with daily awakenings or when patients show signs of clinical improvement allowing cooperation with a more comprehensive examination. If the patient has an unreliable exam and/or is persistently weak, electrophysiological testing (for example, electromyography (EMG) and nerve conduction studies (NCS)) and/or muscle biopsy may be considered [8].

\section{Risk factors and prevention of ICUAW}

In the last decade, studies have identified a number of risk factors for ICUAW, but most are small, observational studies with important methodological limitations $[9,12]$. There are limited and conflicting data regarding the association between severity of illness and ICUAW [9]. Indeed, critical illness neuromyopathy (an important cause of ICUAW) itself is a form of neuromuscular organ failure and similar attention should be placed on prevention and recovery, much the same way that we would approach acute kidney or lung injury.

Hyperglycemia may be an important risk factor for the development of ICUAW. Post hoc analyses of two large randomized control trials looking at intensive insulin therapy in both surgical and medical ICUs reported a significant reduction in ICUAW with tight glycemic control $[13,14]$. However, the results of these secondary analyses and the safety of intensive insulin therapy remains controversial and clinicians should be cautious in using tight glycemic control for the prevention of ICUAW.

There remains substantial controversy regarding the association of ICUAW with two other commonly cited risk factors: corticosteroids and neuromuscular blocking agents (NMBA). Although three prospective observational studies have shown increased risk of ICUAW with corticosteroid exposure $[1,15,16]$, a number of studies have also failed to show a significant association $[12,17]$. Similarly, despite early evidence to suggest persistent weakness after prolonged infusion of vecuronium [18], subsequent studies evaluating NMBAs have not supported any significant association with ICUAW. Importantly, a recent trial of patients with early acute respiratory distress syndrome (ARDS) randomized to cisatricurium demonstrated a significant reduction in 28-day mortality, without any significant difference in ICUAW as compared to the placebo group [19]. In the end, the decision to use corticosteroids and/or NMBA requires a case-by-case review of the potential benefits and risks, and clinicians must be aware of all the evidence in order to make an informed decision.

\section{Barriers to early mobility in the ICU}

In recent years, a number of studies have documented the safety and feasibility of early mobility in the ICU [4-6]. In addition, early rehabilitation is associated with important reductions in delirium, duration of mechanical ventilation, and improved physical function at hospital discharge [20]. Despite this, only about $25 \%$ of all ICU patients receive early therapy [21]. A number of important barriers, both real and perceived, to implementing early rehabilitation at the patient (for example, delirium, hemodynamic instability), provider (for example, staff discomfort, decreased awareness about the importance of early mobility), and institutional (for example, lack of facilities, personnel, equipment) levels have been identified [21,22]. Commonly cited reason for not receiving therapy include oversedation or coma, lack of available rehabilitation staff, and some potentially avoidable including inappropriate vascular access positions, conflict with another planned procedure, 
and poor sedation management and agitation [21]. While simply pairing physical therapy sessions with sedative interruption may enhance the delivery of early rehabilitation, ultimately, overcoming these barriers will require the creation of an ICU culture that prioritizes early rehabilitation through interdisciplinary coordination, communication, and teamwork. Educational strategies focused on the complications of oversedation (for example, bed rest/ immobility, delirium) and its effects on both the shortterm and long-term outcomes (for example, ICUAW, neurocognitive/neuropsychiatric morbidity) may help to facilitate culture change. These elements are essential in ensuring the successful and sustained implementation of such a complex intervention. Finally, the barriers, facilitators, and efficacy of early rehabilitation have been evaluated primarily in medical ICU patients; the applicability of these practices in other ICUs (for example, neurologic, trauma, pediatric) require exploration in future clinical trials.

\section{Early rehabilitation in the ICU: future directions}

Novel rehabilitation technology, such as neuromuscular electrical stimulation (NMES) and cycle ergometry, may provide an opportunity for early rehabilitation even when the patient is unable to actively participate (for example, during acute phase of critical illness). In healthy volunteers, NMES can improve or preserve muscle strength by preventing disuse atrophy through stimulated muscle contraction [23], but there are limited data in the critically ill. In a study of mechanically ventilated patients with chronic obstructive pulmonary disease (COPD), those randomized to NMES with physical therapy had improved muscle strength at 28 days and decreased number of days needed to transfer from bed to chair as compared to physical therapy alone [24]. Cycle ergometry, another novel rehabilitation tool can provide passive, active-assisted, or active range of motion exercises [23]. In a recent study of ninety critically ill patients, those randomized to cycle ergometry showed improvements in quadriceps muscle function, 6-minute walk distance, and self-reported physical functioning at hospital discharge [25]. These promising results require confirmation in large, prospective clinical trials.

Enforced bedrest, oversedation, and delirium are common barriers to early rehabilitation in the ICU. Sedation and analgesia are commonly instituted (with or without neuromuscular blockade) in the ICU for management of patient discomfort, anxiety, and asynchrony during mechanical ventilation. Changes in sedation strategies, including novel sedative agents such as dexmedetomidine [26], use of intermittent sedation [27], or no sedation [28] may help to limit oversedation and delirium in the ICU and improve patient wakefulness and availability for physical therapy.
Even more radical might be finding a viable alternative to mechanical ventilation in which sedation and analgesia use can truly be minimized. Extracorporeal membrane oxygenation (ECMO) has become an area of increasing interest following its successful use for H1N1-associated ARDS [29]. By providing extracorporeal gas exchange support, ECMO may mitigate the need for any aggressive mechanical ventilation in patients with respiratory failure. Indeed, a number of groups have exploited this technology to facilitate rehabilitation and ambulation in ICU patients awaiting lung transplantation $[30,31]$. As this technology continues to improve and miniaturize, it is conceivable that mechanical ventilation could eventually be replaced by ECMO, allowing our critically ill patients to be awake, calm, cooperative, and mobile.

\section{Conclusions}

ICUAW is an important complication that contributes to functional disability and decreased quality of life in ICU survivors. Indeed, it is an important component of the post-intensive care syndrome (PCIS) representing any new or worsening impairment in physical, cognitive, or mental heath status following an acute care hospitalization [32]. The lack of an accepted taxonomy and diagnostic criteria for ICUAW has made it difficult to interpret and compare results across studies. Early rehabilitation may be an important preventative therapy for ICUAW, but there are many important barriers at the patient, provider, and organizational levels which need to be recognized and overcome. Novel rehabilitation (for example, NMES, cycle ergometry) technology may facilitate rehabilitation in patients who cannot actively participate in therapy during the acute phase of their illness. Finally, ECMO may obviate the need for heavy sedation/analgesia and mechanical ventilation, providing a means to provide early rehabilitation to patients with even the most severe forms of respiratory failure in the ICU. The combination of: (1) a coordinated interdisciplinary team; (2) novel advances in both ICU and rehabilitation technology; and (3) a culture that prioritizes early rehabilitation, will help our ICU patients to be awake, calm, cooperative, and mobile, and hopefully translate into substantial improvements in both their short-term and long-term outcomes.

\footnotetext{
Acknowledgements

EF is supported by a Fellowship Award from the Canadian Institutes of Health Research (CIHR). The funding body had no role in the design; in the collection, analysis, and interpretation of data; in the writing of the manuscript; and in the decision to submit the manuscript for publication.
}

\section{Author details}

${ }^{1}$ Interdepartmental Division of Critical Care Medicine, University of Toronto, and the Division of Respirology, Department of Medicine, Mount Sinai Hospital and University Health Network, Toronto, Ontario, Canada. ${ }^{2}$ Mount Sinai Hospital, 600 University Avenue, Suite 18-201, Toronto, Ontario, Canada. 


\section{Authors' contributions}

CML made a substantial contribution to the conception of the mini-review, drafting of the manuscript and revising it critically for important intellectual content. EF conceived the mini-review, and helped in drafting the manuscript and revising it critically for important intellectual content. Both authors read and approved the final manuscript.

\section{Competing interests}

The authors declare that they have no competing interests.

Received: 11 July 2012 Accepted: 3 October 2012

Published: 3 October 2012

\section{References}

1. Herridge MS, Cheung AM, Tansey CM, Matte-Martyn A, Diaz-Granados N Al-Saidi F, Cooper AB, Guest CB, Mazer CD, Mehta S, Stewart TE, Barr A, Cook D, Slutsky AS, Canadian Critical Care Trials Group: One-year outcomes in survivors of the acute respiratory distress syndrome. $N$ Engl J Med 2003, 348:683-693.

2. Herridge MS, Tansey CM, Matté A, Tomlinson G, Diaz-Granados N, Cooper A, Guest CB, Mazer CD, Mehta S, Stewart TE, Kudlow P, Cook D, Slutsky AS, Cheung AM, Canadian Critical Care Trials Group: Functional disability 5 years after acute respiratory distress syndrome. $N$ Engl I Med 2011, 364:1293-1304

3. Fan E, Zanni JM, Dennison CR, Lepre SJ, Needham DM: Critical illness neuromyopathy and muscle weakness in patients in the intensive care unit. AACN Adv Crit Care 2009, 20:243-253.

4. Bailey P, Thomsen GE, Spuhler VJ, Blair R, Jewkes J, Bezdjian L, Veale K Rodriquez L, Hopkins RO: Early activity is feasible and safe in respiratory failure patients. Crit Care Med 2007, 35:139-145.

5. Morris PE, Goad A, Thompson C, Taylor K, Harry B, Passmore L, Ross A, Anderson L, Baker S, Sanchez M, Penley L, Howard A, Dixon L, Leach S, Small R, Hite RD, Haponik E: Early intensive care unit mobility therapy in the treatment of acute respiratory failure. Crit Care Med 2008, $36: 2238-2243$

6. Schweickert WD, Pohlman MC, Pohlman AS, Nigos C, Pawlik AJ, Esbrook CL, Spears L, Miller M, Franczyk M, Deprizio D, Schmidt GA, Bowman A, Barr R, McCallister KE, Hall JB, Kress JP: Early physical and occupational therapy in mechanically ventilated, critically ill patients: a randomised controlled trial. Lancet 2009, 373:1874-1882.

7. Bolton CF, Gilbert JJ, Hahn AF, Sibbald WJ: Polyneuropathy in critically ill patients. I Neurol Neurosurg Psychiatr 1984, 47:1223-1231.

8. Stevens RD, Marshall SA, Cornblath DR, Hoke A, Needham DM, de Jonghe B, Ali NA, Sharshar T: A framework for diagnosing and classifying intensive care unit-acquired weakness. Crit Care Med 2009, 37:S299-308.

9. Fan E: Critical illness neuromyopathy and the role of physical therapy and rehabilitation in critically ill patients. Respir Care 2012, 57:933-946.

10. Bloch S, Polkey MI, Griffiths M, Kemp P: Molecular mechanisms of intensive care unit-acquired weakness. Eur Respir J 2012, 39:1000-1011.

11. De Jonghe B, Cook D, Sharshar T, Lefaucheur JP, Carlet J, Outin H: Acquired neuromuscular disorders in critically ill patients: a systematic review. Intensive Care Med 1998, 24:1242-1250.

12. Stevens RD, Dowdy DW, Michaels RK, Mendez-Tellez PA, Pronovost PJ, Needham DM: Neuromuscular dysfunction acquired in critical illness: a systematic review. Intensive Care Med 2007, 33:1876-1891.

13. van den Berghe G, Wilmer A, Hermans G, Meersseman W, Wouters PJ, Milants I, van Wijngaerden E, Bobbaers H, Bouillon R: Intensive insulin therapy in the medical ICU. N Engl J Med 2006, 354:449-461.

14. van den Berghe G, Wouters P, Weekers F, Verwaest C, Bruyninckx F, Schetz M, Vlasselaers D, Ferdinande P, Lauwers P, Bouillon R: Intensive insulin therapy in critically ill patients. N Engl J Med 2001, 345:1359-1367.

15. De Jonghe B, Sharshar T, Lefaucheur JP, Authier FJ, Durand-Zaleski I, Boussarsar M, Cerf C, Renaud E, Mesrati F, Carlet J, Raphaël JC, Outin H, Bastuji-Garin S, Groupe de Réflexion et d'Etude des Neuromyopathies en Réanimation: Paresis acquired in the intensive care unit. A prospective multicenter study. JAMA 2002, 288:2859-2867.

16. Campellone JV, Lacomis D, Kramer DJ, van Cott AC, Giuliani MJ: Acute myopathy after liver transplantation. Neurology 1998, 50:46-53.

17. Hough CL, Steinberg KP, Thompson BT, Rubenfeld GD, Hudson LD: Intensive care unit-acquired neuromyopathy and corticosteroids in survivors of persistent ARDS. Intensive Care Med 2009, 35:63-68.
18. Segredo V, Caldwell JE, Matthay MA, Sharma ML, Gruenke LD, Miller RD: Persistent paralysis in critically ill patients after long-term administration of vecuronium. N Engl J Med 1992, 327:524-528.

19. Papazian L, Forel J-M, Gacouin A, Penot-Ragon C, Perrin G, Loundou A, Jaber S, Arnal J-M, Perez D, Seghboyan J-M, Constantin J-M, Courant P, Lefrant J-Y, Guérin C, Prat G, Morange S, Roch A: Neuromuscular blockers in early acute respiratory distress syndrome. N Engl I Med 2010, 363:1107-1116.

20. Schweickert WD, Pohlman MC, Pohlman AS, Nigos C, Pawlik AJ, Esbrook CL, Spears L, Miller M, Franczyk M, Deprizio D, Schmidt GA, Bowman A, Barr R, McCallister KE, Hall JB, Kress JP: Early physical and occupational therapy in mechanically ventilated, critically ill patients: a randomized controlled trial. Lancet 2009, 373:1874-1882.

21. Zanni JM, Korupolu R, Fan E, Pradhan P, Janjua K, Palmer JB, Brower RG, Needham DM: Rehabilitation therapy and outcomes in acute respiratory failure: an observational pilot project. J Crit Care 2010, 25:254-262.

22. Pohlman MC, Schweickert WD, Pohlman AS, Nigos C, Pawlik AJ, Esbrook CL, Spears L, Miller M, Franczyk M, Deprizio D, Schmidt GA, Bowman A, Barr R, Mccallister K, Hall JB, Kress JP: Feasibility of physical and occupational therapy beginning from initiation of mechanical ventilation. Crit Care Med 2010, 38:2089-2094.

23. Needham DM, Truong AD, Fan E: Technology to enhance physical rehabilitation of critically ill patients. Crit Care Med 2009, 37:S436-41

24. Zanotti E, Felicetti G, Maini M, Fracchia C: Peripheral muscle strength training in bed-bound patients with COPD receiving mechanical ventilation: effect of electrical stimulation. Chest 2003, 124:292-296.

25. Burtin C, Clerckx B, Robbeets C, Ferdinande P, Langer D, Troosters T, Hermans G, Decramer M, Gosselink R: Early exercise in critically ill patients enhances short-term functional recovery. Crit Care Med 2009, 37:2499-2505.

26. Riker RR, Shehabi Y, Bokesch PM, Ceraso D, Wisemandle W, Koura F, Whitten P, Margolis BD, Byrne DW, Ely EW, Rocha MG, SEDCOM (Safety and Efficacy of Dexmedetomidine Compared With Midazolam) Study Group: Dexmedetomidine vs midazolam for sedation of critically ill patients. JAMA 2009, 301:489-499.

27. Treggiari MM, Romand J-A, Yanez ND, Deem SA, Goldberg J, Hudson L, Heidegger C-P, Weiss NS: Randomized trial of light versus deep sedation on mental health after critical illness. Crit Care Med 2009, 37:2527-2534.

28. Strøm T, Martinussen T, Toft P: A protocol of no sedation for critically ill patients receiving mechanical ventilation: a randomised trial. Lancet 2010, 375:475-480

29. The Australia and New Zealand Extracorporeal Membrane Oxygenation ANZ ECMO Influenza Investigators: Extracorporeal membrane oxygenation for 2009 influenza $A(\mathrm{H} 1 \mathrm{~N} 1)$ acute respiratory distress syndrome. JAMA 2009, 302:1888-1895.

30. Turner DA, Cheifetz IM, Rehder KJ, Williford WL, Bonadonna D, Banuelos SJ, Peterson-Carmichael S, Lin SS, Davis RD, Zaas D: Active rehabilitation and physical therapy during extracorporeal membrane oxygenation while awaiting lung transplantation: a practical approach. Crit Care Med 2011, 39:2593-2598.

31. Fuehner T, Kuehn C, Hadem J, Wiesner O, Gottlieb J, Tudorache I, Olsson KM, Greer M, Sommer W, Welte T, Haverich A, Hoeper MM, Warnecke $\mathrm{G}$ : Extracorporeal membrane oxygenation in awake patients as bridge to lung transplantation. Am J Respir Crit Care Med 2012, 185:763-768.

32. Needham DM, Davidson J, Cohen H, Hopkins RO, Weinert C, Wunsch H, Zawistowski C, Bemis-Dougherty A, Berney SC, Bienvenu OJ, Brady SL, Brodsky MB, Denehy L, Elliott D, Flatley C, Harabin AL, Jones C, Louis D, Meltzer W, Muldoon SR, Palmer JB, Perme C, Robinson M, Schmidt DM, Scruth E, Spill GR, Storey CP, Render M, Votto J, Harvey MA: Improving long-term outcomes after discharge from intensive care unit: report from a stakeholders' conference. Crit Care Med 2012, 40:502.

\section{Pre-publication history}

The pre-publication history for this paper can be accessed here: http://www.biomedcentral.com/1741-7015/10/115/prepub

doi:10.1186/1741-7015-10-115

Cite this article as: Lee and Fan: ICU-acquired weakness: what is preventing its rehabilitation in critically ill patients? BMC Medicine 2012 10:115. 